\title{
Analysis of Security-Reliability Trade-off for Multi-hop Cognitive Relaying Protocol with TAS/SC Technique
}

\author{
Pham Minh Nam*, Phu Tran Tin
}

Faculty of Electronics Technology, Industrial University of Ho Chi Minh City, Ho Chi Minh City 700000, Vietnam

\begin{tabular}{l} 
A R T I C L E I N F O \\
\hline Article history: \\
Received: 29 June, 2020 \\
Accepted: 11 August, 2020 \\
Online: 08 September, 2020 \\
\hline Keywords: \\
Intercept Probability \\
Multi-hop Relaying Network \\
Outage Probability \\
Physical-layer Security \\
Selection combining (SC) \\
Transmit antenna selection (TAS) \\
Underlay Cognitive Radio \\
\end{tabular}

\begin{abstract}
A B S T R A C T
This paper studies a trade-off between security (intercept probability (IP)) and reliability (outage probability (OP)) for a multi-hop decode-and-forward (DF) relaying protocol in an underlay cognitive radio network, in presence of a multi-antenna eavesdropper. In the considered protocol, all primary and secondary terminals are equipped with multiple antennas, and they employ transmit antenna selection (TAS) (at transmitter sides) and selection combining (SC) (at receiver sides) techniques to enhance the system performance. Relying on channel state information (CSI) of the primary-to-secondary interference links known or unknown, two efficient TAS/SC techniques are proposed for the secondary networks. Moreover, operating on the underlay spectrum sharing mode, the secondary transmitters including source and relays must adapt their transmit power so that OP of the primary network is not higher than a pre-determined threshold. Under impact of Rayleigh fading and co-channel interference, the end-to-end (e2e) OP and IP are expressed by exact closed-form expressions that are verified by Monte-Carlo simulations. Then, both simulation and theoretical results are presented to show the OP-IP trade-off.
\end{abstract}

\section{Introduction}

MIMO (Multiple Input Multiple Output) [1, 2] and diversity relaying [3-4] are efficient techniques used for wireless communication systems operating on interference, path-loss, fading, noise environment. In MIMO, transmitters and receivers are equipped with multiple antennas, and use transmit and receive diversity techniques to enhance data rate, channel capacity, diversity order as well as to reduce outage probability (OP), error rates. Efficient combiners such as SC (Selection Combining) [5, 6], EGC (Equal-Gain Combining) [7], MRC (Maximal Ratio Combining) [8] are commonly used by the receivers to enhance reliability of the data decoding. The best combiner is MRC, but its implementation is too difficult, while the SC combiner is simplest. Indeed, using SC, the receivers only use the receive antenna which provides the highest signal-to-interference plus noise ratio (SINR) to decode the received signals. In transmit diversity techniques, transmit antenna selection (TAS) $[9,10]$ and maximal ratio transmission (MRT) [11] are often used. In TAS, the transmitters select one of their antennas to send data to the receivers. To obtain the highest SINR, TAS requires feedback channel state information (CSI) from the receivers for selecting the best transmit antenna. Unlike TAS, the receivers employing MRT use all the

\footnotetext{
*Corresponding Author: Pham Minh Nam, phamminhnam@iuh.edu.vn
}

antennas to transmit data. The implementation of MRT is hence more difficult than that of TAS, but it obtains higher performance. To further enhance the system performances for wireless communication systems, combination between the transmit diversity and receiver diversity techniques, such as TAS/SC [12], TAS/MRC [12], MRT/SC [13], MRT/MRC [14], were proposed.

Relaying techniques can be efficiently used when the distance between a source and a destination is enough far or a source node cannot directly communicate with a destination node. In these techniques, intermediate nodes or relay nodes are employed to help the source-destination data transmission [3-4, 15-16]. Depends on the signal processing methods, the relays are categorized into two main groups: DF (decode and forward) [15-16] and AF (amplify and forward) [17-18]. The DF relays first decode the received data, encode it again, before sending the encoded signal to the destination. On the contrary, the AF relays only forward the amplified signals to the destination without performing the decoding. Hence, the AF technique is simpler than the DF one, but DF outperforms AF because the noises can be removed at the DF relays. Until now, published works concerned with performance evaluation of the relaying scenarios have mainly focused on dualhop networks, e.g. [3-4, 15-18]. In [19-20], the authors considered multi-hop relaying models, where the source data are relayed to 
the destination over multi-hop or multi-relay. Reference [19] evaluated end-to-end (e2e) symbol error rate of the multi-hop AF relaying protocol. In [20], the multi-hop DF relaying protocol was proposed and analyzed. To enhance the e2e system performances for the multi-hop relaying networks over fading environments, various diversity relaying methods were proposed. References [20, 21 introduced path-selection strategies to exploit spatial diversity. The authors in $[22,23]$ studied the e2e OP of multi-hop MIMO relaying protocols where the TAS/MRC technique was used to obtain diversity gain at each hop.

In wireless communication systems, security is a critical issue because the transmitted data can be easily overheard by eavesdroppers. Recently, physical-layer security (PLS) [24, 25] has gained much attention as a promising solution to guarantee secure communication. PLS can be simply implemented in practice because the security can be attained, relying on physical channel information such as link distances and channel quality. Indeed, the data transmission can be considered to be secure if the data channel is better than the eavesdropping one. The secrecy performances of the PLS systems can be evaluated via secrecy capacity defined as difference of channel capacity obtained on the data and eavesdropping links. References [26-29] proposed the transmit and receive diversity techniques to enhance secrecy performances for the MIMO systems. In [30-31], secure communication relaying protocols operating over fading channels were proposed and analyzed. Conventionally, the source and relays cooperate together to confuse the eavesdropper, i.e., they randomly generate code-books as transmitting the data [31]. Reference [32] analyzed the e2e secrecy performance of the multihop DF relaying protocol over Nakagami- $m$ fading channels with imperfect transceiver hardware. Unlike [26-32], performance metrics of the PLS systems considered in $[33,34]$ are OP of the data transmission and intercept probability (IP) of the eavesdroppers. As shown in [33, 34], trade-off between IP and OP was investigated.

Due to rapid increasing of wireless devices, J. Mitola proposed cognitive radio (CR) [35] to solve spectrum scarcity issue. In CR, primary users can share their licensed bands to secondary users provided that the primary QoS is guaranteed. To realize this, the secondary users have to adjust their transmit power appropriately so that co-channel interference at the primary receivers must be below a given threshold [36, 37]. Recently, PLS in CR [38, 39] has been much attention of researchers. Reference [40] investigated trade-off between IP and OP of the secure communication in the secondary network operating over Rician fading channels. Moreover, in [40], the TAS/SC technique is used for the primary data transmission. The authors in [41] proposed a cooperative routing approach to enhance the e2e secrecy performances for the underlay CR networks, as compared with a conventional multi-hop relaying method. In [42], the TAS/SC technique is used at each hop in the secondary network to obtain better secrecy performance in presence of hardware imperfection. In [43], a best-path selection strategy was proposed for secrecy performance enhancement of cluster-based CR networks using cooperative jamming technique.

This paper investigates the e2e IP and OP performance of the multi-hop MIMO relaying protocol in the underlay CR network with presence of a secondary multi-antenna eavesdropper. In the proposed scheme, the TAS/SC technique is used by both the primary and secondary networks. More particularly, the secondary source and relays must reduce their transmit power to guarantee that $\mathrm{OP}$ at the primary receiver is always below a required threshold. Under the joint impact of the limited transmit power and co-channel interference, the secondary transmitters use TAS/SC at each hop to improve the reliability of the data transmission. Also in the secondary network, the eavesdropper with the SC combiner attempts to illegally decode the transmitted data over multiple hops. Moreover, we consider two cases where the interference from the primary transmitter on the secondary nodes is known or not. From two these cases, we propose different TAS/SC techniques for the secondary network. Different with $[40,43]$ in which the wireless devices only have single antenna; all the nodes in our proposed protocol have multiple antennas, and employ TAS/SC to attain higher diversity order. While references [41-44] derived the secrecy performance by using secrecy capacity, this paper investigates the trade-off between IP and OP. Moreover, references $[42,43]$ do not consider the presence of the primary transmitter, as well as ignore the impact of the co-channel interference from the primary network.

In the following, we summarize the main contribution of this paper as

- We propose TAS/SC-based multi-hop MIMO relaying protocols in PLS underlay CR networks. Moreover, under impact of the co-channel interference, we consider two efficient TAS/SC techniques applied for the secondary network, depending on channel state information (CSI) between the secondary nodes and the primary transmitter is known or not.

- From an exact closed-form formula of the outage performance of the primary network, we derive expressions of the transmit power for the secondary transmitters including source and relays.

- Exact closed-form expressions of the e2e OP and IP over Rayleigh fading channel are derived, and are validated by Monte-Carlo simulations.

This paper is organized into sections as follows. Section 2 describes system model and operation principle of the proposed model. Section 3 measures the system performances in terms of IP and OP. Both simulation results and analytical results are presented in Section 4. Finally, conclusions are provided in Section 5.

\section{System Model}

In Fig. 1, the primary transmitter $(\mathrm{T})$ and the primary receiver (R) are equipped $N_{\mathrm{T}} \quad N_{\mathrm{R}}$ antennas, respectively, and they use $\mathrm{TAS} / \mathrm{SC}$ to communicate with each other. Considering the secondary network; the source node $\mathrm{S}_{0}$ wants to sends its data to the destination $\mathrm{S}_{K}$ via $(K-1)$ intermediate relays named $\mathrm{S}_{1}$, $\mathrm{S}_{2}, \ldots, \mathrm{S}_{K-1}$. Assume that all of the nodes $\mathrm{S}_{k}(k=0,1, \ldots, K)$ have $N_{\mathrm{S}}$ antennas, and the $\mathrm{S}_{0} \rightarrow \mathrm{S}_{K}$ data transmission is accomplished via $K$ orthogonal time slots. In addition, at the $k-$ th hop, $\mathrm{S}_{k-1}$ sends the data to $\mathrm{S}_{k}$ by performing TAS/SC. Also in the secondary 
network, an $N_{\mathrm{E}}$-antenna eavesdropper (E) attempts to overhear the data transmitted at each hop.
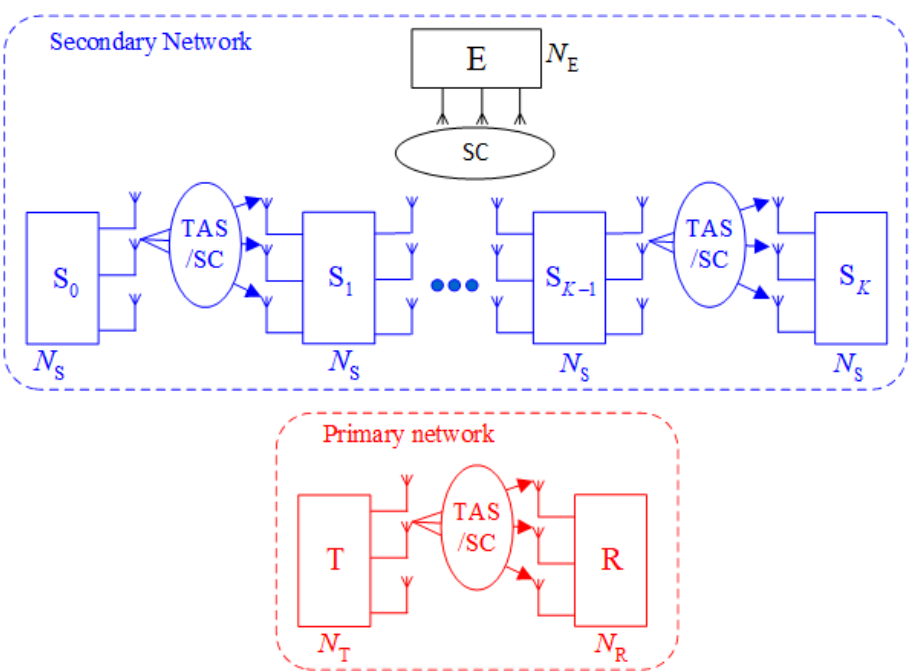

Figure 1: System model of the proposed TAS/SC-based multi-hop relaying protocol in the underlay CR networks.

We denote $\gamma_{\mathrm{XY}}$ as channel gain of the $\mathrm{X}-\mathrm{Y}$ Rayleigh fading channel, and hence the channel gain is an exponential random variable $(\mathrm{RV})$. Similar to [3], parameter of $\gamma_{\mathrm{XY}}$ is $\lambda_{\mathrm{XY}}=d_{\mathrm{XY}}^{\beta}$, where $d_{\mathrm{XY}}$ is $\mathrm{X}-\mathrm{Y}$ distance, and $\beta$ is path-loss exponent. More particularly, distribution functions of $\gamma_{\mathrm{XY}}$ are given as follows:

$$
F_{\gamma_{\mathrm{XY}}}(x)=1-\exp \left(-\lambda_{\mathrm{XY}} x\right), f_{\gamma_{\mathrm{XY}}}(x)=\lambda_{\mathrm{XY}} \exp \left(-\lambda_{\mathrm{XY}} x\right),
$$

where $F_{U}($.$) and f_{U}($.$) are \mathrm{CDF}$ (cumulative distribution function) and PDF (probability density function) of $\operatorname{RV} U$, respectively.

Next, we consider the primary and secondary data transmission at the $k$-th time slot, in which $\mathrm{S}_{k-1}$ and $\mathrm{T}$ at the same time send their data to $\mathrm{S}_{k}$ and $\mathrm{R}$, respectively. Assume that $\mathrm{S}_{k-1}$ uses the $m$-th transmit antenna, $\mathrm{T}$ uses the $p$-th the antenna, $\mathrm{S}_{k}$ uses the $n$-th receive antenna and $\mathrm{R}$ uses the $q$ - th receive antenna, where $m \in\left\{1,2, \ldots, N_{\mathrm{S}}\right\}, n \in\left\{1,2, \ldots, N_{\mathrm{S}}\right\}, \quad p \in\left\{1,2, \ldots, N_{\mathrm{T}}\right\}$, $q \in\left\{1,2, \ldots, N_{\mathrm{R}}\right\}$. Due to the cross co-channel interference between two networks, the instantaneous SINR obtained at $\mathrm{S}_{k}$ and $\mathrm{R}$ can be formulated by (2) and (3), respectively as

$$
\begin{aligned}
\varphi_{\mathrm{T}^{p} \mathrm{R}^{q}} & =\frac{P_{\mathrm{T}} \gamma_{\mathrm{T}^{p} \mathrm{R}^{q}}}{P_{\mathrm{S}_{k-1}} \gamma_{\mathrm{S}_{k-1}^{m} \mathrm{R}^{q}}+\sigma_{0}^{2}}, \\
\varphi_{\mathrm{S}_{k-1}^{m} \mathrm{~S}_{k}^{n}} & =\frac{P_{\mathrm{S}_{k-1}} \gamma_{\mathrm{S}_{k-1}^{m} \mathrm{~S}_{k}^{n}}}{P_{\mathrm{T}} \gamma_{\mathrm{T}^{p} \mathrm{~S}_{k}^{n}}+\sigma_{0}^{2}} .
\end{aligned}
$$

Now, we introduce notations used in (2)-(3). $P_{\mathrm{T}}$ is transmit power of each antenna of T. $\sigma_{0}^{2}$ is variance of Gaussian noises at
$\mathrm{R}$, and it is also variance at all the receivers. $P_{\mathrm{S}_{k-1}}$ is transmit power of $\mathrm{S}_{k-1} \cdot \gamma_{\mathrm{X}^{a} \mathrm{Y}^{b}}$ is channel gain between the $a$ - th antenna of $X$ and the $b-$ th antenna of $Y$, where $(X, Y) \in\left\{\mathrm{S}_{k-1}, \mathrm{~S}_{k}, \mathrm{~T}, \mathrm{R}\right\}$ and $(a, b) \in\{m, n, p, q\}$.

Considering the $\mathrm{T} \rightarrow \mathrm{R}$ transmission; the $\mathrm{TAS} / \mathrm{SC}$ technique is used to obtain the maximum SINR between $\mathrm{T}$ and $\mathrm{R}$, as

$$
\varphi_{\mathrm{T}^{u} \mathrm{R}^{v}}=\max _{p=1,2, \ldots, N_{\mathrm{T}}}\left(\max _{q=1,2, \ldots, N_{\mathrm{R}}}\left(\varphi_{\mathrm{T}^{p} \mathrm{R}^{q}}\right)\right),
$$

where $u$ and $v$ are the optimal transmit-receive pair, $u \in\left\{1,2, \ldots, N_{\mathrm{T}}\right\}, v \in\left\{1,2, \ldots, N_{\mathrm{R}}\right\}$.

Comment 1: To realize the strategy given in (4), the $\mathrm{T}$ and $\mathrm{R}$ nodes have to know the interference component $P_{\mathrm{S}_{k-1}} \gamma_{\mathrm{S}_{k-1}^{m} \mathrm{R}^{q}}$ which can be obtained by requesting the cooperation from $\mathrm{S}_{k-1}$.

\subsection{OP of Primary Network}

From (4), the instantaneous channel capacity of the T-R link is given as

$$
C_{\mathrm{P}, k}=\frac{1}{K} \log _{2}\left(1+\varphi_{\mathrm{T}^{u} \mathrm{R}^{v}}\right)
$$

where the fraction $1 / K$ implies that the data transmission in the secondary network is realized in $K$ orthogonal time slots.

Next, OP of the primary network is defined as the probability that $C_{\mathrm{P}, k}$ is below a positive value denoted by $C_{\mathrm{Pth}}$. Then, combining (2), (4) and (5), we can formulate OP at R as

$$
\begin{aligned}
\mathrm{OP}_{\mathrm{P}, k} & =\operatorname{Pr}\left(C_{\mathrm{P}, k}<C_{\mathrm{Pth}}\right)=\operatorname{Pr}\left(\varphi_{\mathrm{T}^{u} \mathrm{R}^{v}}<\rho_{\mathrm{Pth}}\right) \\
& =\prod_{p=1}^{N_{\mathrm{T}}} \prod_{q=1}^{N_{\mathrm{R}}} \underbrace{\operatorname{Pr}\left(\frac{P_{\mathrm{T}} \gamma_{\mathrm{T}^{p} \mathrm{R}^{q}}}{P_{\mathrm{S}_{k-1}} \gamma_{\mathrm{S}_{k-1}^{m} \mathrm{R}^{q}}+\sigma_{0}^{2}}<\rho_{\mathrm{Pth}}\right)}_{I_{1}},
\end{aligned}
$$

where

$$
\rho_{\text {Pth }}=2^{K C_{\text {Pth }}}-1 \text {. }
$$

Next, we rewrite the probability $I_{1}$ marked in (6) as

$$
\begin{aligned}
I_{1} & =\operatorname{Pr}\left(P_{\mathrm{T}} \gamma_{\mathrm{T}^{p} \mathrm{R}^{q}}<P_{\mathrm{S}_{k-1}} \rho_{\text {Pth }} \gamma_{\mathrm{S}_{k-1}^{m} \mathrm{R}^{q}}+\sigma_{0}^{2} \rho_{\text {Pth }}\right) \\
& =\int_{0}^{+\infty} F_{\gamma_{\mathrm{T}^{p} \mathrm{R}^{q}}}\left(\mu_{1} P_{\mathrm{S}_{k-1}} x+\mu_{2}\right) f_{\gamma_{\mathrm{S}_{k-1}^{m} \mathrm{R}^{q}}}(x) d x,
\end{aligned}
$$

where

$$
\mu_{1}=\frac{\rho_{\mathrm{Pth}}}{P_{\mathrm{T}}}, \mu_{2}=\frac{\sigma_{0}^{2} \rho_{\mathrm{Pth}}}{P_{\mathrm{T}}} .
$$


Substituting $\mathrm{CDF} \quad F_{\gamma_{\mathrm{T}} \mathrm{R}^{q}}\left(\mu_{1} P_{\mathrm{S}_{k-1}} x+\mu_{2}\right)$ and $\mathrm{PDF}$ $f_{\gamma_{\mathrm{S}_{k-1}^{m} \mathrm{R}^{q}}}(x)$ obtained in (1) into (8); which yields

$$
\begin{aligned}
I_{1}=1- & \lambda_{\mathrm{S}_{k-1} \mathrm{R}} \exp \left(-\lambda_{\mathrm{TR}} \mu_{2}\right) \\
& \quad \times \int_{0}^{+\infty} \exp \left(-\lambda_{\mathrm{TR}} P_{\mathrm{S}_{k-1}} \mu_{1} x\right) \exp \left(-\lambda_{\mathrm{S}_{k-1} \mathrm{R}} x\right) d x \\
=1- & \frac{\lambda_{\mathrm{S}_{k-1} \mathrm{R}}}{\lambda_{\mathrm{S}_{k-1} \mathrm{R}}+\lambda_{\mathrm{TR}} P_{\mathrm{S}_{k-1}} \mu_{1}} \exp \left(-\lambda_{\mathrm{TR}} \mu_{2}\right) .
\end{aligned}
$$

Then, substituting (10) into (6), we obtain

$$
\mathrm{OP}_{\mathrm{P}, k}=\left[1-\frac{\lambda_{\mathrm{S}_{k-1} \mathrm{R}}}{\lambda_{\mathrm{S}_{k-1} \mathrm{R}}+\lambda_{\mathrm{TR}} P_{\mathrm{S}_{k-1}} \mu_{1}} \exp \left(-\lambda_{\mathrm{TR}} \mu_{2}\right)\right]^{N_{\mathrm{T}} N_{\mathrm{R}}} .
$$

\subsection{Transmit Power of Secondary Transmitters}

At first, the primary QoS is defined as $\mathrm{OP}_{\mathrm{P}, k} \leq \varepsilon_{\mathrm{Pth}}(\forall k)$, where $\varepsilon_{\mathrm{Pth}}$ is a predefined threshold. By solving $\mathrm{OP}_{\mathrm{P}, k}=\varepsilon_{\mathrm{Pth}}$, an exact closed-form expression of $P_{\mathrm{S}_{k-1}}$ can be given as

$$
P_{\mathrm{S}_{k-1}}=\left[\left(\frac{\exp \left(-\lambda_{\mathrm{TR}} \mu_{2}\right)}{1-\left(\varepsilon_{\mathrm{Pth}}\right) \frac{1}{N_{\mathrm{T}} N_{\mathrm{R}}}}-1\right) \frac{\lambda_{\mathrm{S}_{k-1} \mathrm{R}}}{\lambda_{\mathrm{TR}} \mu_{1}}\right]^{+},
$$

where $[x]^{+}=\max (0, x)$.

At high transmit power values of $P_{\mathrm{T}}$, i.e., $P_{\mathrm{T}} \rightarrow+\infty$, we can approximate $P_{\mathrm{S}_{k-1}}$ as in (13):

$$
P_{\mathrm{S}_{k-1}} \stackrel{P_{\mathrm{T}} \rightarrow+\infty}{\approx}\left(\frac{1}{1-\left(\varepsilon_{\mathrm{Pth}}\right) \frac{1}{N_{\mathrm{T}} N_{\mathrm{R}}}}-1\right) \frac{\lambda_{\mathrm{S}_{k-1} \mathrm{R}} P_{\mathrm{T}}}{\lambda_{\mathrm{TR}} \rho_{\mathrm{Pth}}}=\xi P_{\mathrm{T}},
$$

where

$$
\xi=\left(\frac{1}{1-\left(\varepsilon_{\mathrm{Pth}}\right) \frac{1}{N_{\mathrm{T}} N_{\mathrm{R}}}}-1\right) \frac{\lambda_{\mathrm{S}_{k-1} \mathrm{R}}}{\lambda_{\mathrm{TR}} \rho_{\mathrm{Pth}}} .
$$

Comment 2: The transmit power of the secondary transmitters is calculated by the primary network as in (12), and then sent to the $\mathrm{S}_{k-1}$ node. Next, it is worth noting that $P_{\mathrm{S}_{k-1}}=0$ means the primary network does not obtain the required QoS, i.e., $\mathrm{OP}_{\mathrm{P}, k}>\varepsilon_{\mathrm{Pth}}$. Finally, equation (13) shows that as $P_{\mathrm{T}}$ is high enough, the primary QoS is always satisfied, and the transmit power $P_{\mathrm{S}_{k-1}}$ becomes a linear function of $P_{\mathrm{T}}$.

\subsection{TAS/SC methods}

In this section, two practical $\mathrm{TAS} / \mathrm{SC}$ techniques are considered. The TAS/SC technique is proposed in [12], in which the transmitter and receiver select an optimal transmit-receive antennas to maximize the instantaneous SINR of the data link. However, with presence of the co-channel interference, the $\mathrm{TAS} / \mathrm{SC}$ technique in [12] may not be optimal any more. This motivates us to reconsider the TAS/SC technique in the interference environment.

\section{Case 1: Co-channel interference from $P_{\mathrm{T}}$ is known}

In this case, we assume that the co-channel interference can be perfectly estimated by the secondary nodes. Hence, similar to (4), the TAS/SC technique can be set up by $\mathrm{S}_{k-1}$ and $\mathrm{S}_{k}$ as

$$
\varphi_{\mathrm{S}_{k-1}^{z} \mathrm{~S}_{k}^{t}}=\max _{m=1,2, \ldots, N_{\mathrm{S}}}\left(\max _{n=1,2, \ldots, N_{\mathrm{S}}}\left(\frac{P_{\mathrm{S}_{k-1}} \gamma_{\mathrm{S}_{k-1}^{m} \mathrm{~S}_{k}^{n}}}{P_{\mathrm{T}} \gamma_{\mathrm{T}^{u} \mathrm{~S}_{k}^{n}}+\sigma_{0}^{2}}\right)\right),
$$

where $z$ and $t$ are the selected transmit-receive antenna pair at $\mathrm{S}_{k-1}$ and $\mathrm{S}_{k}$, respectively, $(z, t) \in\left\{1,2, \ldots, N_{\mathrm{S}}\right\}$.

For a fair comparison, assume that the interference from the primary network is also known by $\mathrm{E}$. With the SC combiner, the SINR obtained at $\mathrm{E}$ can be given as

$$
\varphi_{\mathrm{S}_{k-1}^{z} \mathrm{E}^{e}}=\max _{r=1,2, \ldots, N_{\mathrm{E}}}\left(\frac{P_{\mathrm{S}_{k-1}} \gamma_{\mathrm{S}_{k-1}^{z} \mathrm{E}^{r}}}{P_{\mathrm{T}} \gamma_{\mathrm{T}^{u} \mathrm{E}^{r}}+\sigma_{0}^{2}}\right),
$$

where $e$ is the selected antennas at $\mathrm{E}$ for decoding the source data, and $e \in\left\{1,2, \ldots, N_{\mathrm{E}}\right\}$.

Case 2: Co-channel interference from $P_{\mathrm{T}}$ is unknown

It is worth noting that in practice the primary network does not need to cooperate with the secondary network (but the secondary network must cooperate with the primary network). Hence, the secondary nodes may not obtain perfectly the information about the co-channel interference caused by PT. In this case, the TAS/SC technique should be performed, only relying on CSIs of the data links, i.e.

$$
\gamma_{\mathrm{S}_{k-1}^{a} \mathrm{~S}_{k}^{b}}=\max _{m=1,2, \ldots, N_{\mathrm{S}}}\left(\max _{n=1,2, \ldots, N_{\mathrm{S}}}\left(\gamma_{\mathrm{S}_{k-1}^{m} \mathrm{~S}_{k}^{n}}\right)\right),
$$

where $a$ and $b$ are the chosen antennas at $\mathrm{S}_{k-1}$ and $\mathrm{S}_{k}$, respectively, $(a, b) \in\left\{1,2, \ldots, N_{\mathrm{S}}\right\}$.

We can observe that the TAS/SC technique in (17) is only suboptimal because the $\mathrm{S}_{k-1}$ and $\mathrm{S}_{k}$ nodes have not perfect information of the channel gain $\gamma_{\mathrm{T}^{u} \mathrm{~S}_{k}^{n}}$.

Hence, the SINR obtained at $\mathrm{S}_{k}$ in this case can be given as

$$
\varphi_{\mathrm{S}_{k-1}^{a} \mathrm{~S}_{k}^{b}}=\frac{P_{\mathrm{S}_{k-1}} \gamma_{\mathrm{S}_{k-1}^{a} \mathrm{~S}_{k}^{b}}}{P_{\mathrm{T}} \gamma_{\mathrm{T}^{u} \mathrm{~S}_{k}^{b}}+\sigma_{0}^{2}} .
$$


Also, it is assumed that $\mathrm{E}$ has no information of the co-channel interference links, and hence the best antenna selected by $\mathrm{E}$ is given as

$$
\gamma_{\mathrm{S}_{k-1}^{a} \mathrm{E}^{l}}=\max _{r=1,2, \ldots, N_{\mathrm{E}}}\left(\gamma_{\mathrm{S}_{k-1}^{z} \mathrm{E}^{r}}\right)
$$

where $l\left(l \in\left\{1,2, \ldots, N_{\mathrm{E}}\right\}\right)$ is the selected antennas at $\mathrm{E}$ for decoding the source data. Then, we can formulate the SINR obtained at $\mathrm{E}$ as

$$
\varphi_{\mathrm{S}_{k-1}^{a} \mathrm{E}^{l}}=\frac{P_{\mathrm{S}_{k-1}} \gamma_{\mathrm{S}_{k-1}^{a} \mathrm{E}^{l}}}{P_{\mathrm{T}} \gamma_{\mathrm{T}^{u} \mathrm{E}^{e}}+\sigma_{0}^{2}} .
$$

At high transmit power $P_{\mathrm{T}}$, i.e. $P_{\mathrm{T}} \rightarrow+\infty, P_{\mathrm{T}} \square \sigma_{0}^{2}$, from (13), equations (15), (16), (18) and (20) can be approximated respectively as

$$
\begin{aligned}
& \varphi_{\mathrm{S}_{k-1}^{z} \mathrm{~S}_{k}^{t}} \stackrel{P_{\mathrm{T}} \rightarrow+\infty}{\approx} \max _{m=1,2, \ldots, N_{\mathrm{S}}}\left(\max _{n=1,2, \ldots, N_{\mathrm{S}}}\left(\frac{\xi \gamma_{\mathrm{S}_{k-1}^{m} \mathrm{~S}_{k}^{n}}}{\gamma_{\mathrm{T}^{u} \mathrm{~S}_{k}^{n}}}\right)\right), \\
& \varphi_{\mathrm{S}_{k-1}^{z} \mathrm{E}^{e}} \stackrel{P_{\mathrm{T}} \rightarrow+\infty}{\approx} \max _{r=1,2, \ldots, N_{\mathrm{E}}}\left(\frac{\xi \gamma_{\mathrm{S}_{k-1}^{z} \mathrm{E}^{r}}}{\gamma_{\mathrm{T}^{u} \mathrm{E}^{r}}}\right) \text {, } \\
& \varphi_{\mathrm{S}_{k-1}^{a} \mathrm{~S}_{k}^{b}} \stackrel{P_{\mathrm{T}} \rightarrow+\infty}{\approx} \frac{\xi \gamma_{\mathrm{S}_{k-1}^{a} \mathrm{~S}_{k}^{b}}}{\gamma_{\mathrm{T}^{u} \mathrm{~S}_{k}^{b}}}, \\
& \varphi_{\mathrm{S}_{k-1}^{a} \mathrm{E}^{l}} \stackrel{P_{\mathrm{T}} \rightarrow+\infty}{\approx} \frac{\xi \gamma_{\mathrm{S}_{k-1}^{z} \mathrm{E}^{l}}}{\gamma_{\mathrm{T}^{u} \mathrm{E}^{e}}} .
\end{aligned}
$$

Comment 3: As observed from (21)-(24), at high $P_{\mathrm{T}}$ values, the obtained SINRs do not depend on $P_{\mathrm{T}}$.

\subsection{E2e Channel Capacity of Data and Eavesdropping Links}

Due to usage of the DF relaying technique, the e2e channel capacity of the $\mathrm{S}_{0} \rightarrow \mathrm{S}_{K}$ link in the first and second cases can be respectively given as (see $[19,29])$

$$
\begin{gathered}
C_{\mathrm{D}, \mathrm{e} 2 \mathrm{e}}^{\text {Cae } 1}=\frac{1}{K} \log _{2}\left(1+\min _{k=1,2, \ldots, K}\left(\varphi_{\mathrm{S}_{k-1}^{z} \mathrm{~S}_{k}^{t}}\right)\right), \\
C_{\mathrm{D}, \mathrm{e} 2 \mathrm{e}}^{\text {Case 2 }}=\frac{1}{K} \log _{2}\left(1+\min _{k=1,2, \ldots, K}\left(\varphi_{\mathrm{S}_{k-1}^{a} \mathrm{~S}_{k}^{b}}\right)\right) .
\end{gathered}
$$

Considering the channel capacity obtained at the eavesdropper. Because of the random code-book generation at each hop, the channel capacity at E in Case 1 and Case 2 can be formulated, respectively as

$$
\begin{gathered}
C_{\mathrm{E}, \mathrm{e} 2 \mathrm{e}}^{\text {Case } 1}=\frac{1}{K} \log _{2}\left(1+\max _{k=1,2, \ldots, K}\left(\varphi_{\mathrm{S}_{k-1}^{z} \mathrm{E}^{e}}\right)\right), \\
C_{\mathrm{E}, \mathrm{e} 2 \mathrm{e}}^{\text {Case } 2}=\frac{1}{K} \log _{2}\left(1+\max _{k=1,2, \ldots, K}\left(\varphi_{\mathrm{S}_{k-1}^{a} \mathrm{E}^{l}}\right)\right) .
\end{gathered}
$$

\section{Evaluation of E2e OP and IP}

At first, the e2e OP and IP are respectively defined as in [36]:

$$
\begin{gathered}
\mathrm{OP}_{\mathrm{e} 2 \mathrm{e}}^{\text {Case } i}=\operatorname{Pr}\left(C_{\mathrm{D}, \mathrm{e} 2 \mathrm{e}}^{\text {Case } i}<C_{\mathrm{Sth}}\right), \\
\mathrm{IP}_{\mathrm{e} 2 \mathrm{e}}^{\text {Case } i}=\operatorname{Pr}\left(C_{\mathrm{E}, \mathrm{e} 2 \mathrm{e}}^{\text {Case } i} \geq C_{\mathrm{Sth}}\right),
\end{gathered}
$$

where $C_{\mathrm{Sth}}$ is a pre-determined threshold, $i \in\{1,2\}$.

\subsection{E2e $O P$}

In this sub-section, we exactly and asymptotically evaluate the e2e OP in two cases considered in Sub-section 2.3. At first, combining (25), (26) and (29), we can obtain (31) as

$$
\begin{aligned}
\mathrm{OP}_{\mathrm{e} 2 \mathrm{e}}^{\text {Case } i} & =\operatorname{Pr}\left(\min _{k=1,2, \ldots, K}\left(\varphi_{\mathrm{S}_{k-1}^{x} \mathrm{~S}_{k}^{y}}\right)<\rho_{\mathrm{Sth}}\right) \\
& =1-\prod_{k=1}^{K}\left(1-\operatorname{Pr}\left(\varphi_{\mathrm{S}_{k-1}^{x} \mathrm{~S}_{k}^{y}}<\rho_{\mathrm{Sth}}\right)\right) \\
& =1-\prod_{k=1}^{K}\left(1-\mathrm{OP}_{k}^{\text {Case } i}\right),
\end{aligned}
$$

where

$$
\begin{aligned}
& \rho_{\text {Sth }}=2^{K C_{\mathrm{Sth}}}-1, \mathrm{OP}_{k}^{\text {Case } i}=\operatorname{Pr}\left(\varphi_{\mathrm{S}_{k-1}^{x} \mathrm{~S}_{k}^{y}}<\rho_{\mathrm{Sth}}\right), \\
& (x, y)=\left\{\begin{array}{l}
(z, t), \text { if } i=1 \\
(a, b), \text { if } i=2
\end{array}\right.
\end{aligned}
$$

Case 1: Co-channel interference from $P_{\mathrm{T}}$ is known

Substituting (15) into $\mathrm{OP}_{k}^{\text {Case } 1}$, which yields

$$
\begin{aligned}
\mathrm{OP}_{k}^{\text {Case } 1} & =\prod_{m=1}^{N_{\mathrm{S}}} \prod_{n=1}^{N_{\mathrm{S}}} \operatorname{Pr}\left(\frac{P_{\mathrm{S}_{k-1}} \gamma_{\mathrm{S}_{k-1}^{m} \mathrm{~S}_{k}^{n}}}{P_{\mathrm{T}_{\mathrm{T}} \gamma_{\mathrm{S}}^{n}}+\sigma_{0}^{2}}<\rho_{\mathrm{Sth}}\right) \\
& =\prod_{m=1}^{N_{\mathrm{S}}} \prod_{n=1}^{N_{\mathrm{S}}} \operatorname{Pr}\left(\gamma_{\mathrm{S}_{k-1}^{m} \mathrm{~S}_{k}^{n}}<\mu_{3} \gamma_{\mathrm{T}^{u} \mathrm{~S}_{k}^{n}}+\mu_{4}\right) \\
& =\left[\int_{0}^{+\infty} F_{\gamma_{\mathrm{S}_{k-1}^{m} \mathrm{~S}_{k}^{n}}}\left(\mu_{3} x+\mu_{4}\right) f_{\gamma_{\mathrm{T}^{u} \mathrm{~S}_{k}^{n}}}(x) d x\right]^{N_{\mathrm{S}}^{2}},
\end{aligned}
$$

where

$$
\mu_{3}=\frac{\rho_{\mathrm{Sth}} P_{\mathrm{T}}}{P_{\mathrm{S}_{k-1}}}, \mu_{4}=\frac{\rho_{\mathrm{Sth}} \sigma_{0}^{2}}{P_{\mathrm{S}_{k-1}}}
$$

Similar to the derivation steps in (8)-(10); substituting CDF $F_{\gamma_{\mathrm{s}_{k-1}^{m} \mathrm{~s}_{k}^{n}}}\left(\mu_{3} x+\mu_{4}\right)$ and PDF $f_{\gamma_{\mathrm{T}^{u} \mathrm{~s}_{k}^{n}}}(x)$ obtained in (1) into (33), after some manipulation, we can obtain (35) as follows:

$$
\mathrm{OP}_{k}^{\text {Case 1 }}=\left[1-\frac{\lambda_{\mathrm{TS}_{k}}}{\lambda_{\mathrm{TS}_{k}}+\lambda_{\mathrm{S}_{k-1} \mathrm{~S}_{k}} \mu_{3}} \exp \left(-\lambda_{\mathrm{S}_{k-1} \mathrm{~S}_{k}} \mu_{4}\right)\right]^{N_{\mathrm{S}}^{2}} .
$$

Substituting (35) into (31), an exact closed-form formula of the e2e OP in the first case can be written as 


$$
\begin{aligned}
& \mathrm{OP}_{\mathrm{e} 2 \mathrm{e}}^{\text {Case } 1}= \\
& 1-\prod_{k=1}^{K}\left\{1-\left[1-\frac{\lambda_{\mathrm{TS}_{k}}}{\lambda_{\mathrm{TS}_{k}}+\lambda_{\mathrm{S}_{k-1} \mathrm{~S}_{k}} \mu_{3}} \exp \left(-\lambda_{\mathrm{S}_{k-1} \mathrm{~S}_{k}} \mu_{4}\right)\right]^{N_{\mathrm{S}}^{2}}\right\},
\end{aligned}
$$

At high $P_{\mathrm{T}}$ values, by using the approximate expression of $\varphi_{\mathrm{S}_{k-1}^{z} \mathrm{~S}_{k}^{t}}$ in (21), with the same derivation method, $\mathrm{OP}_{\mathrm{e} 2 \mathrm{e}}^{\text {Case } 1}$ can be approximated as follows:

$$
\mathrm{OP}_{\mathrm{e} 2 \mathrm{e}}^{\text {Case } 1} \stackrel{P_{\mathrm{T}} \rightarrow+\infty}{\approx} 1-\prod_{k=1}^{K}\left[1-\left(\frac{\lambda_{\mathrm{S}_{k-1} \mathrm{~S}_{k}} \mu_{3}}{\lambda_{\mathrm{TS}_{k}}+\lambda_{\mathrm{S}_{k-1} \mathrm{~S}_{k}} \mu_{3}}\right)^{N_{\mathrm{S}}^{2}}\right]
$$

Case 2: Co-channel interference from $P_{\mathrm{T}}$ is unknown

Substituting (18) into $\mathrm{OP}_{k}^{\text {Case } 2}$, we can obtain (38) as

$$
\mathrm{OP}_{k}^{\text {Case 2 }}=\int_{0}^{+\infty} F_{\gamma_{\mathrm{s}_{k-1}^{a} \mathrm{~s}_{k}^{b}}}\left(\mu_{3} x+\mu_{4}\right) f_{\gamma_{\mathrm{T}^{u} \mathrm{~s}_{k}^{b}}}(x) d x
$$

From (17), we can write $F_{\gamma_{\mathrm{s}_{k-1}^{a} \mathrm{l}_{k}^{b}}}\left(\mu_{3} x+\mu_{4}\right)$ as

$$
\begin{aligned}
& F_{\gamma_{\mathrm{S}_{k-1}^{a} b_{k}^{b}}}\left(\mu_{3} x+\mu_{4}\right)=\prod_{m=1}^{N_{\mathrm{S}}} \prod_{n=1}^{N_{\mathrm{S}}} F_{\gamma_{\mathrm{S}_{k-1}^{m} \mathrm{~S}_{k}^{n}}}\left(\mu_{3} x+\mu_{4}\right) \\
& =\left(1-\exp \left(-\lambda_{\mathrm{S}_{k-1} \mathrm{~S}_{k}}\left(\mu_{3} x+\mu_{4}\right)\right)\right)^{N_{\mathrm{S}}^{2}} \\
& =1+\sum_{m=1}^{N_{\mathrm{S}}^{2}}(-1)^{m} C_{N_{\mathrm{S}}^{2}}^{m} \exp \left(-m \lambda_{\mathrm{S}_{k-1} \mathrm{~S}_{k}} \mu_{4}\right) \exp \left(-m \lambda_{\mathrm{S}_{k-1} \mathrm{~S}_{k}} \mu_{3} x\right),
\end{aligned}
$$

where $C_{N_{\mathrm{S}}^{2}}^{m}$ is binomial coefficient which is expressed as

$$
C_{N_{\mathrm{S}}^{2}}^{m}=\frac{\left(N_{S}^{2}\right) !}{m !\left(N_{S}^{2}-m\right) !}
$$

Substituting (39) and PDF $f_{\gamma_{\mathrm{T}^{u} \mathrm{~s}_{k}^{b}}}(x)$ into (38), after some manipulation, we obtain

$$
\mathrm{OP}_{k}^{\text {Case 2 }}=1+\sum_{m=1}^{N_{\mathrm{S}}^{2}}(-1)^{m} \frac{C_{N_{\mathrm{S}}^{2}}^{m} \lambda_{\mathrm{TS}_{k}} \exp \left(-m \lambda_{\mathrm{S}_{k-1} \mathrm{~S}_{k}} \mu_{4}\right)}{\lambda_{\mathrm{TS}_{k}}+m \lambda_{\mathrm{S}_{k-1} \mathrm{~S}_{k}} \mu_{3}} .
$$

Hence, the e2e OP in this case can be expressed as

$$
\mathrm{OP}_{\mathrm{e} 2 \mathrm{e}}^{\text {Case 2 }}=1-\prod_{k=1}^{K}\left[\sum_{m=1}^{N_{\mathrm{S}}^{2}} \frac{(-1)^{m+1} C_{N_{\mathrm{S}}^{2}}^{m} \lambda_{\mathrm{TS}_{k}} \exp \left(-m \lambda_{\mathrm{S}_{k-1} \mathrm{~S}_{k}} \mu_{4}\right)}{\lambda_{\mathrm{TS}_{k}}+m \lambda_{\mathrm{S}_{k-1} \mathrm{~S}_{k}} \mu_{3}}\right] \text {. }
$$

As $P_{\mathrm{T}}$ is high enough, $\mathrm{OP}_{\mathrm{e} 2 \mathrm{e}}^{\text {Case } 2}$ can be approximated as

$$
\mathrm{OP}_{\mathrm{e} 2 \mathrm{e}}^{\text {Case } 2} \stackrel{P_{\mathrm{T}} \rightarrow+\infty}{\approx} 1-\prod_{k=1}^{K}\left[\sum_{m=1}^{N_{\mathrm{S}}^{2}}(-1)^{m+1} \frac{C_{N_{\mathrm{S}}^{2}}^{m} \lambda_{\mathrm{TS}_{k}}}{\lambda_{\mathrm{TS}_{k}}+\lambda_{\mathrm{S}_{k-1} \mathrm{~S}_{k}} \mu_{3}}\right] .
$$

\subsection{E2e IP}

Combining (27), (28) and (30), which yields

$$
\begin{aligned}
\mathrm{IP}_{\mathrm{e} 2 \mathrm{e}}^{\text {Case } i} & =\operatorname{Pr}\left(\max _{k=1,2, \ldots, K}\left(\varphi_{\mathrm{S}_{k-1}^{x} \mathrm{E}^{y}}\right) \geq \rho_{\mathrm{Sth}}\right) \\
& =1-\prod_{k=1}^{K}\left(\operatorname{Pr}\left(\varphi_{\mathrm{S}_{k-1}^{x} \mathrm{E}^{y}}<\rho_{\mathrm{Sth}}\right)\right),
\end{aligned}
$$

where

$$
(x, y)=\left\{\begin{array}{l}
(z, e), \text { if } i=1 \\
(a, l), \text { if } i=2
\end{array}\right.
$$

With the same derivation methods of $\mathrm{OP}_{\mathrm{e} 2 \mathrm{e}}^{\mathrm{Case} 1}$ and $\mathrm{OP}_{\mathrm{e} 2 \mathrm{e}}^{\mathrm{Case} 2}$, we can calculate $\operatorname{Pr}\left(\varphi_{\mathrm{S}_{k-1}^{x} \mathrm{E}^{y}}<\rho_{\mathrm{Sth}}\right)$, and then $\mathrm{IP}_{\mathrm{e} 2 \mathrm{e}}^{\text {Case } 1}$ and $\mathrm{IP}_{\mathrm{e} 2 \mathrm{e}}^{\text {Case } 2}$ can be respectively written as in (46) and (47):

$$
\begin{gathered}
\mathrm{IP}_{\mathrm{e} 2 \mathrm{e}}^{\mathrm{Case} 1}=1-\prod_{k=1}^{K}\left\{\left[1-\frac{\lambda_{\mathrm{TE}} \exp \left(-\lambda_{\mathrm{S}_{k-1} \mathrm{E}} \mu_{4}\right)}{\lambda_{\mathrm{TE}}+\lambda_{\mathrm{S}_{k-1}} \mu_{3}}\right]^{N_{\mathrm{E}}}\right\}, \\
\mathrm{IP}_{\mathrm{e} 2 \mathrm{e}}^{\text {Case 2 }}=1-\prod_{k=1}^{K}\left[1+\sum_{n=1}^{N_{\mathrm{E}}}(-1)^{n} \frac{C_{N_{\mathrm{E}}}^{n} \lambda_{\mathrm{TE}} \exp \left(-n \lambda_{\mathrm{S}_{k-1} \mathrm{E}} \mu_{4}\right)}{\lambda_{\mathrm{TE}}+n \lambda_{\mathrm{S}_{k-1}} \mu_{3}}\right] .
\end{gathered}
$$

At high $P_{\mathrm{T}}$ regions, (46) and (47) can be approximated by

$$
\begin{gathered}
\mathrm{IP}_{\mathrm{e} 2 \mathrm{e}}^{\text {Case } 1} \stackrel{P_{\mathrm{T}} \rightarrow+\infty}{\approx} 1-\prod_{k=1}^{K}\left(\frac{\lambda_{\mathrm{S}_{k-1} \mathrm{E}} \mu_{3}}{\lambda_{\mathrm{TE}}+\lambda_{\mathrm{S}_{k-1} \mathrm{E}} \mu_{3}}\right)^{N_{\mathrm{E}}}, \\
\mathrm{IP}_{\mathrm{e} 2 \mathrm{e}}^{\text {Case } 2} \stackrel{P_{\mathrm{T}} \rightarrow+\infty}{\approx} 1-\prod_{k=1}^{K}\left[1+\sum_{n=1}^{N_{\mathrm{E}}}(-1)^{n} \frac{C_{N_{\mathrm{E}}}^{n} \lambda_{\mathrm{TE}}}{\lambda_{\mathrm{TE}}+n \lambda_{\mathrm{S}_{k-1} \mathrm{E}} \mu_{3}}\right] .
\end{gathered}
$$

\section{Simulation Results}

Section 4 verifies the formulas obtained in Section 3 by MonteCarlo simulations realized by MATLAB. Assume that the nodes T, $\mathrm{R}, \mathrm{S}_{k}$, and $\mathrm{E}$ are located at $\left(x_{\mathrm{T}}, y_{\mathrm{T}}\right),\left(x_{\mathrm{R}}, y_{\mathrm{R}}\right),(k / K, 0)$, and $\left(x_{\mathrm{E}}\right.$, $\left.y_{\mathrm{E}}\right)$, respectively. In all the simulations, the system parameters are set as follows: $x_{\mathrm{E}}=0.5, y_{\mathrm{E}}=0.3$, and the by $\beta=3, \sigma_{0}^{2}=1$, $C_{\text {Pth }}=1.5, C_{\text {Sth }}=0.2, N_{\mathrm{S}}=N_{\mathrm{E}}=2, \varepsilon_{\mathrm{P}}=0.01$.

\subsection{OP of Primary Network and Transmit Power of Secondary Transmitters} $P_{\mathrm{T}}$

Figure 2 presents $\mathrm{OP}$ of the primary network as a function of

$$
0.5,0.6) \text { and }(0.35,0.3
$$


$N_{\mathrm{T}}=N_{\mathrm{R}}=1$, OP at $\mathrm{T}$ is higher than $\varepsilon_{\mathrm{P}}$ when $P_{\mathrm{T}}<15 \mathrm{~dB}$, and when $N_{\mathrm{T}}=N_{\mathrm{R}}=2$, OP at $\mathrm{T}$ is higher than $\varepsilon_{\mathrm{P}}$ when $P_{\mathrm{T}}<0 \mathrm{~dB}$. This means than the primary network can obtain the required QoS with lower transmit power $P_{\mathrm{T}}$ by equipping more antennas at $\mathrm{T}$ and $\mathrm{R}$. It is shown in this figure that the OP value converges to $\varepsilon_{\mathrm{P}}$ at high $P_{\mathrm{T}}$ value due to the data transmission of the secondary networks. Finally, we can observe from Fig. 2 that the simulation results verify the theoretical ones.

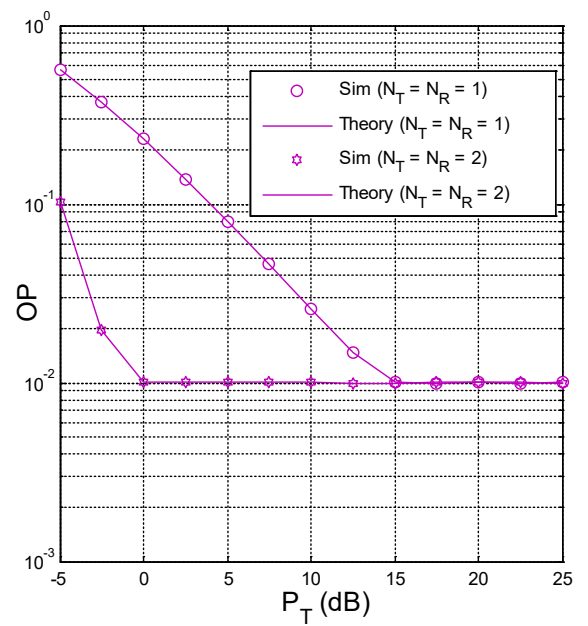

Figure 2: $\mathrm{OP}$ of the primary network as a function of $P_{\mathrm{T}}(\mathrm{dB})$ when $x_{\mathrm{T}}=0.5$, $y_{\mathrm{T}}=0.6, x_{\mathrm{R}}=0.35, y_{\mathrm{R}}=0.3, K=2$.

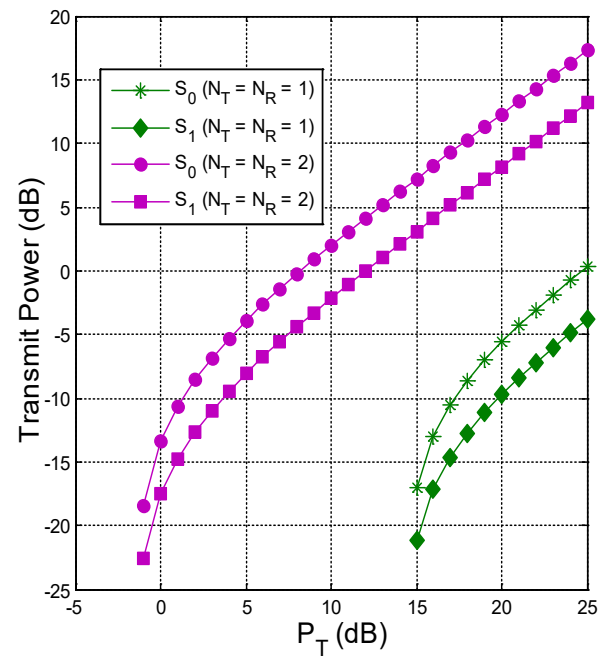

Figure 3: Transmit power of the secondary transmitters as a function of $P_{\mathrm{T}}(\mathrm{dB})$

$$
\text { when } x_{\mathrm{T}}=0.5, y_{\mathrm{T}}=0.6, x_{\mathrm{R}}=0.35, y_{\mathrm{R}}=0.3, K=2 \text {. }
$$

Figure 3 illustrates the transmit power of $\mathrm{S}_{0}$ and $\mathrm{S}_{1}$ as a function of $P_{\mathrm{T}}$ in $\mathrm{dB}$. We note that the system parameters in Fig. 3 are same with those in Fig. 2. As shown in Fig. 3, the secondary transmitters $\mathrm{S}_{0}$ and $\mathrm{S}_{1}$ can use the licensed bands when $P_{\mathrm{T}}$ is high enough, i.e., when $N_{\mathrm{T}}=N_{\mathrm{R}}=1$ then $P_{\mathrm{T}} \geq 15 \mathrm{~dB}$, and when
$N_{\mathrm{T}}=N_{\mathrm{R}}=2$ then $P_{\mathrm{T}} \geq-1 \mathrm{~dB}$. Because the OP performance of the primary network is better when the $\mathrm{T}$ and $\mathrm{R}$ nodes are equipped with more antennas, the secondary network has more opportunity to access the licensed bands. Finally, we can see that the transmit power of $S_{0}$ is higher than that of $S_{1}$ because the $S_{0}-R$ distance is higher than the $S_{1}-R$ one.

\subsection{OP and IP of Secondary Network}

In this sub-section, we present the e2e OP and IP of the proposed protocol in two cases considered in Section 3. In all of the presented figures, we fix the number of antennas at $\mathrm{T}$ and $\mathrm{R}$ by $2\left(N_{\mathrm{T}}=N_{\mathrm{R}}=2\right)$, and the positions of $\mathrm{T}$ and $\mathrm{R}$ at $(0.5,0.6)$ and $(0.5,0.4)$, respectively.

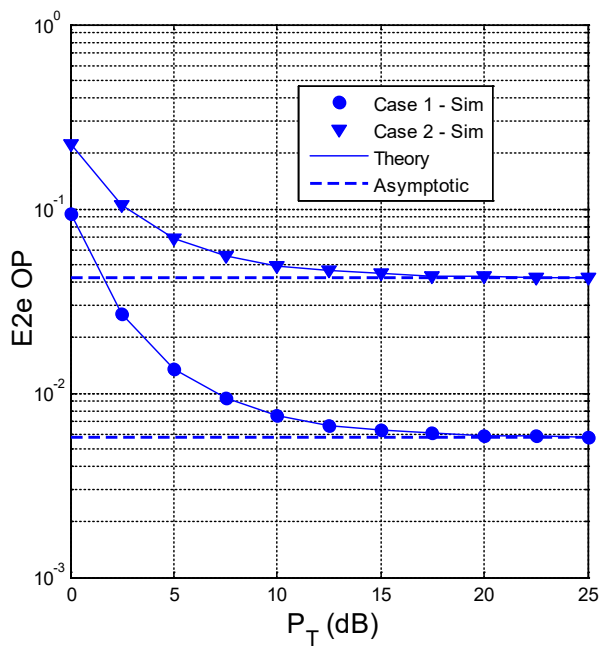

Figure 4: E2e OP as a function of $P_{\mathrm{T}}(\mathrm{dB})$ when $x_{\mathrm{T}}=0.5, y_{\mathrm{T}}=0.6, x_{\mathrm{R}}=0.5$, $y_{\mathrm{R}}=0.4, N_{\mathrm{T}}=N_{\mathrm{R}}=2, K=3$.

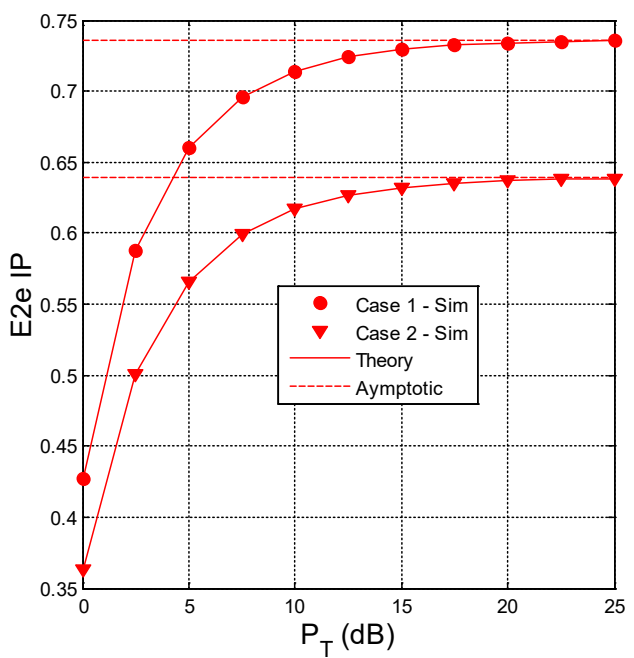

Figure 5: E2e IP as a function of $P_{\mathrm{T}}(\mathrm{dB})$ when $x_{\mathrm{T}}=0.5, y_{\mathrm{T}}=0.6, x_{\mathrm{R}}=0.5$,

$$
y_{\mathrm{R}}=0.4, N_{\mathrm{T}}=N_{\mathrm{R}}=2, K=3 \text {. }
$$


Figures 4 and 5 present $\mathrm{OP}$ and IP as a function of $P_{\mathrm{T}}$ in $\mathrm{dB}$ when the number of hops $(K)$ equals to 3 . As we can see, the OP and IP values at high $P_{\mathrm{T}}$ region do not depend on $P_{\mathrm{T}}$, as proved in Section 3. This also means that with the impact of the co-channel interference from the primary network, the secondary network cannot obtain diversity order, i.e., there exists the error floor in the OP performance. As shown in Fig. 4, the e2e OP in Case 1 is much lower than that in Case 2. However, Figure 5 presents that the e2e IP in Case 1 is higher than that in Case 2. From Figs. 4 and 5, it is clearly presented the trade-off between OP and IP. Particularly, if the transmit power $P_{\mathrm{T}}$ is higher, the e2e OP is lower but the e2e IP is higher. Also, if the TAS/SC technique in Case 1 is used, the OP performance is better, but the IP performance is worse. Finally, it is also illustrated that the simulation results verify the derived formulas of the e2e OP and IP in Section 3.

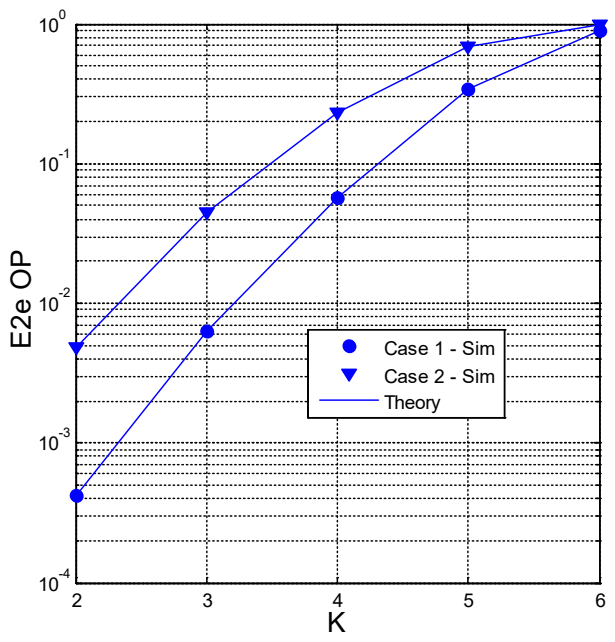

Figure 6: E2e OP as a function of $K$ when $x_{\mathrm{T}}=0.5, y_{\mathrm{T}}=0.6, x_{\mathrm{R}}=0.5$, $y_{\mathrm{R}}=0.4, N_{\mathrm{T}}=N_{\mathrm{R}}=2, P_{\mathrm{T}}=15(\mathrm{~dB})$.

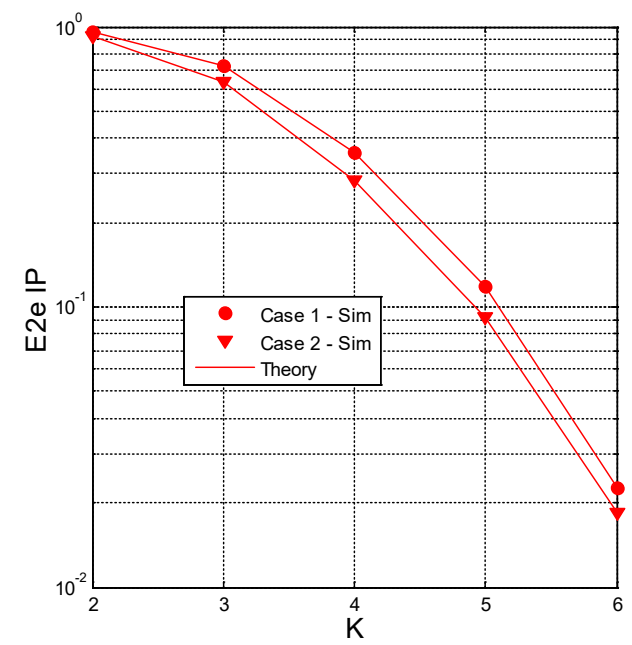

Figure 7: E2e IP as a function of $K$ when $x_{\mathrm{T}}=0.5, y_{\mathrm{T}}=0.6, x_{\mathrm{R}}=0.5$, $y_{\mathrm{R}}=0.4, N_{\mathrm{T}}=N_{\mathrm{R}}=2, P_{\mathrm{T}}=15(\mathrm{~dB})$.
In Figs. 6-7, the e2e OP and IP is presented as a function of the number of hops $(K)$ with $P_{\mathrm{T}}=15(\mathrm{~dB})$. As presented, the OP values increase with the increasing of the number of hops, but the IP values decrease. Similar to Figs. 4-5, the OP and IP values in Case 1 are lower and higher than those in Case 2. Again, it is shown that there exists the trade-off between the e2e OP and IP performance, and the value of $K$ should be carefully designed. For example, if the required OP of the secondary network is 0.1 , then the optimal number of hops used in Case 1 and 2 (to obtain minimum value of IP) is 4 and 3 , respectively.

\section{Conclusion}

This paper proposed the TAS/SC based multi-hop relaying protocol in the PLS underlay CR networks. The practical applications of the proposed protocol are listed as: i) enhancing the spectrum usage efficiency with underlay CR approach; ii) enhancing the performance of the primary and secondary networks with the TAS/SC techniques; iii) two practical TAS/SC techniques are applied for the secondary network; iv) all the derived expressions are in closed-form, which can be easily used for evaluating and optimizing the systems. Moreover, the results showed the trade-off between the IP and OP at the secondary network, and the bad effect of the co-channel interference from the primary network. Finally, the important parameters such as the number of hops and the transmit power of the primary transmitter should be carefully designed.

\section{Conflict of Interest}

The authors declare no conflict of interest.

\section{Acknowledgment}

This research is funded by Vietnam National Foundation for Science and Technology Development (NAFOSTED) under grant number 102.04-2017.317.

\section{References}

[1] L. Liu, R. Chen, S. Geirhofer, K. Sayana, Z. Shi, Y. Zhou, "Downlink MIMO in LTE-advanced: SU-MIMO vs. MU-MIMO” IEEE Commun. Mag., 50(2), 140-147, 2012. https://doi.org/10.1109/MCOM.2012.6146493

[2] Y. Kim, H. Ji, J. Lee, Y.-H. Nam, B. Loong Ng, I. Tzanidis, Y. Li, "Full Dimension MIMO (FD-MIMO): The Next Evolution of MIMO in LTE Systems" IEEE Wireless Commun., 21(3), 92-100, 2014. https://doi.org/10.1109/MWC.2014.6845053

[3] J. N. Laneman, D. N. Tse, G. W. Wornell, "Cooperative Diversity in Wireless Networks: Efficient Protocols and Outage Behavior" IEEE Trans. Inform Theory, 50(12), 3062-3080, 2004. https://doi.org/10.1109/TIT.2004.838089

[4] M. A. Beserra de Melo, D. Benevides da Costa, "An Efficient RelayDestination Selection Scheme for Multiuser Multirelay Downlink Cooperative Networks" IEEE Trans. Veh. Techn., 61(5), 2354 - 2360, 2012. https://doi.org/ 10.1109/TVT.2012.2192488

[5] M. D. Selvaraj, Ranjan K. Mallik , "Performance of Full CSI Selection Combining for Cooperative Diversity Systems" IEEE Trans. Commun., 60(9), 2482 - 2488, 2012. https://doi.org/10.1109/TCOMM.2012.071212.110101

[6] O. S. Badarneh, D. B. da Costa, M. Benjillali and M. Alouini, "Selection Combining Over Double \$ \alpha \$-\$mu\$ Fading Channels," IEEE Trans. Veh. Techn., 69(3), 3444-3448, 2020. https://doi.org/10.1109/TVT.2020.2969224

[7] Y. Song, S.D. Blostein, J. Cheng, "Exact Outage Probability for Equal Gain Combining with Cochannel Interference in Rayleigh Fading" IEEE Trans. $\begin{array}{llll}\text { Wireless } & \text { Commun., } & \text { 2(5), }\end{array}$ https://doi.org/10.1109/TWC.2003.816796

[8] X.W. Cui, Q.T. Zhang, Z.M. Feng, "Outage Probability for Maximal Ratio Combining of Arbitrarily Correlated Faded Signals Corrupted by Multiple Rayleigh Interferers" IEEE Trans. Veh. Techn., 55(1), 383- 386, 2006. https://doi.org/10.1109/TVT.2005.861186 
[9] S. Kim, M. Shin, C. Lee, "Transmit Antenna Selection Scheme for Iterative Receivers in MIMO Systems" IEEE Sig. Proces. Lett., 14(12), 916 - 919, 2007. https://doi.org/10.1109/LSP.2007.906230

[10] S. Kim, "Transmit Antenna Selection for Precoding-Aided Spatial Modulation," IEEE Access, 8, 40723-40731, 2020. https://doi.org/10.1109/ACCESS.2020.2976732

[11] M. K. Arti, M. R. Bhatnagar, "Maximal Ratio Transmission in AF MIMO Relay Systems Over Nakagami- $m$ Fading Channels," IEEE Trans. Veh. Techn., 64(5), 1895-1903, 2015. https://doi.org/10.1109/TVT.2014.2334631

[12] D. T. Hung, T. T. Duy, T. T. Phuong, D. Q. Trinh, T. Hanh, "Performance Comparison between Fountain Codes-Based Secure MIMO Protocols With and Without using Non-Orthogonal Multiple Access" Entropy, 21(10), 928, 2019. https://doi.org/10.3390/e21100982

[13] A. F. Coskun, O. Kucur, "Performance Analysis of Maximal-Ratio Transmission/Receive Antenna Selection in Nakagami- $m$ Fading Channels With Channel Estimation Errors and Feedback Delay" IEEE Trans. Veh. Techn., 61(3), 1099-1108, 2012. https://doi.org/10.1109/TVT.2012.2183650

[14] V. C. Papamichael, P. Karadimas, "Performance Evaluation of Actual Multielement Antenna Systems Under Transmit Antenna Selection/Maximal Ratio Combining," IEEE Antennas Wireless Propag. Lett., 10, 690 - 692, 2011. https://doi.org/10.1109/LAWP.2011.2161662

[15] T. T. Duy, Trung Q. Duong, D.B. da Costa, V.N.Q. Bao, M. Elkashlan, "Proactive Relay Selection with Joint Impact of Hardware Impairment and Co-channel Interference" IEEE Trans. Commun., 63(5), 1594-1606, 2015. https://doi.org/10.1109/TCOMM.2015.2396517

[16] P. N. Son, T. T. Duy, "A New Approach for Two-Way Relaying Networks: Improving Performance by Successive Interference Cancellation, Digital Network Coding and Opportunistic Relay Selection" Wireless Netw., 26(2), 1315-1329, 2020. https://doi.org/10.1007/s11276-019-02186-1

[17] E. Bjornson, M. Matthaiou, M. Debbah, "A New Look at Dual-Hop Relaying: Performance Limits with Hardware Impairments," IEEE Trans. Commun., 61(11), 4512-4525, https://doi.org/10.1109/TCOMM.2013.100913.130282

[18] J. Ma, C. Huang, S. Cui, Q. Li, "Energy Efficiency of Amplify-and-Forward Full-Duplex Relay Channels," IEEE Wireless Commun. Lett., 8(5), 1365 1368, 2019. https://doi.org/10.1109/LWC.2019.2918290

[19] G. Farhadi, N. C. Beaulieu, "A General Framework for Symbol Error Probability Analysis of Wireless Systems and Its Application in Amplify-andForward Multihop Relaying" IEEE Trans. Veh. Techn., 59(3), 1505 - 1511, 2010. https://doi.org/10.1109/TVT.2009.2037642

[20] M. R. Bhatnagar, "Performance Analysis of a Path Selection Scheme in MultiHop Decode-and-Forward Protocol" IEEE Commun. Lett., 16(12), 1980 1983, 2012. https://doi.org/10.1109/TVT.2009.2037642

[21] T. D. Hieu, T. T. Duy, B.-S. Kim, "Performance Enhancement for Multi-hop Harvest-to-Transmit WSNs With Path-Selection Methods in Presence of Eavesdroppers and Hardware Noises" IEEE Sensors Journal, 18(12), 5173 5186, 2018. https://doi.org/10.1109/JSEN.2018.2829145

[22] I. H. Lee, D. Kim, "Outage Probability of Multi-Hop MIMO Relaying With Transmit Antenna Selection and Ideal Relay Gain Over Rayleigh Fading Channels" IEEE Trans. Commun., 57(2), 357 - 360, 2009. https://doi.org/10.1109/TCOMM.2009.02.070058

[23] A. A. AbdelNabi, F. S. Al-Qahtani, M. Shaqfeh, S. S. Ikki, H. M. Alnuweiri, "Performance Analysis of MIMO Multi-Hop System With TAS/MRC in Poisson Field of Interferers," IEEE Trans. Commun., 64(2), 525-540, 2016. https://doi.org/10.1109/TCOMM.2015.2496291

[24] A. D. Wyner, "The Wire-tap Channel" Bell System Technical Journal, 54(8), 1355 - 1387, 1975. https://doi.org/10.1002/j.1538-7305.1975.tb02040.x

[25] I. Csiszar, J. Korner, "Broadcast Channels with Confidential Messages" IEEE Trans. Inform. Theory, 24(3), $339-348,1978$. https://doi.org/10.1109/TIT.1978.1055892

[26] N. S. Ferdinand, D. B. da Costa, A. L. F. de Almeida, M. Latva-aho, "Physical Layer Secrecy Performance of TAS Wiretap Channels with Correlated Main and Eavesdropper Channels" IEEE Wireless Commun. Lett., 3(1), 86-89, 2014. https://doi.org/10.1109/WCL.2013.112313.130733

[27] J. Xiong, Y. Tang, D. Ma, P. Xiao, K. Wong, "Secrecy Performance Analysis for TAS-MRC System With Imperfect Feedback" IEEE Trans. Inf. Forensics $\begin{array}{llll}\text { Security, } & \mathbf{1 0}(8), & 1617-1629, & 2015 .\end{array}$ https://doi.org/10.1109/TIFS.2015.2421358

[28] J. Si, Z. Li, J. Cheng, C. Zhong, "Asymptotic Secrecy Outage Performance for TAS/MRC Over Correlated Nakagami- $m$ Fading Channels," IEEE Trans. $\begin{array}{lll}\text { Commun., } & \text { 67(11), } & \text { 7700-7714, }\end{array}$ https://doi.org/10.1109/TCOMM.2019.2935444

[29] D. Tran, H. Tran, D. Ha, G. Kaddoum, "Secure Transmit Antenna Selection Protocol for MIMO NOMA Networks Over Nakagami-m Channels," IEEE Systems Journal, 14(1), 253-264, 2020. https://doi.org/10.1109/JSYST.2019.2900090

[30] I. Krikidis, "Opportunistic Relay Selection For Cooperative Networks With Secrecy Constraints" IET Commun., 4(15), 1787 - 1791, 2010. https://doi.org/ 10.1049/iet-com.2009.0634

www.astesi.com
[31] J. Mo, M. Tao, Y. Liu, "Relay Placement for Physical Layer Security: A Secure Connection Perspective," IEEE Commun. Lett., 16(6), 878-881, 2012. https://doi.org/10.1109/LCOMM.2012.042312.120582

[32] P. T. Tin, D. T. Hung, T. T. Duy, M. Voznak, "Analysis of Probability of Nonzero Secrecy Capacity for Multi-hop Networks in Presence of Hardware Impairments over Nakagami- $m$ Fading Channels" RadioEngineering, 25(4), 774-782, 2016. http://dx.doi.org/10.13164/re.2016.0774

[33] Y. Zou, B. Champagne, W. P. Zhu, L. Hanzo, "Relay-Selection Improves the Security-Reliability Trade-off in Cognitive Radio Systems," IEEE Trans. $\begin{array}{lllll}\text { Commun., } & \mathbf{6 3}(1), & 215 \quad 228, & - & 2015 .\end{array}$ http://dx.doi.org/10.1109/TCOMM.2014.2377239

[34] G. Zhang, Y. Gao, H. Luo, S. Wang, M. Guo and N. Sha, "Security Performance Analysis for Best Relay Selection in Energy-Harvesting Cooperative Communication Networks," IEEE Access, vol. 8, pp. 26-2020, http://dx.doi.org/10.1109/ACCESS.2019.2960819

[35] J. Mitola, G. Q. Maguire, "Cognitive Radio: Making Software Radios More Personal" IEEE Pers. Commun., 6(4), 13-18, 1999. http://dx.doi.org/10.1109/98.788210

[36] Y. Guo, G. Kang, N. Zhang, W. Zhou, P. Zhang, "Outage Performance of Relay-Assisted Cognitive-Radio System Under Spectrum-Sharing Constraints" Electronics Lett., $\quad \mathbf{4 6}(2), \quad 1-2, \quad 2010$ http://dx.doi.org/10.1049/el.2010.2159

[37] K. Tourki, K. A. Qaraqe, M.-S. Alouini, "Outage Analysis for Underlay Cognitive Networks Using Incremental Regenerative Relaying” IEEE Trans. Veh. Techn., 62(2), 721-734, 2013. http://dx.doi.org/ 10.1109/TVT.2012.2222947

[38] Y. Liu, L. Wang, T. D. Tran, M. Elkashlan, T. Q. Duong, "Relay Selection for Security Enhancement in Cognitive Relay Networks" IEEE Wireless Commun. Lett., 4(1): 46-49, 2015 . http://dx.doi.org/10.1109/LWC.2014.2365808

[39] M. Elkashlan, L. Wang, T. Q Duong, G. K. Karagiannidis, A. Nallanathan, "On the Security of Cognitive Radio Networks" IEEE Trans. Veh. Techn., 64(8), 3790 - 3795, 2015. http://dx.doi.org/10.1109/TVT.2014.2358624

[40] P. M. Nam, T. T. Duy, P. V. Ca, "End-to-end Security-Reliability Analysis of Multi-hop Cognitive Relaying Protocol with TAS/SC-based Primary Communication, Total Interference Constraint and Asymmetric Fading Channels" International Journal of Communication Systems, 32(2), 1-16, 2019. http://dx.doi.org/10.1002/DAC.3854

[41] H. D. Hung, T. T. Duy, M. Voznak, ""Secrecy Outage Performance of Multihop LEACH Networks Using Power Beacon Aided Cooperative Jamming With Jammer Selection Methods," AEU International Journal of Electronics and Communications, 124, 1-24, 2020 https://doi.org/10.1016/j.aeue.2020.153357

[42] P. T. Tin, D. T. Hung, N. N. Tan, T. T. Duy, M. Voznak, "Secrecy Performance Enhancement for Underlay Cognitive Radio Networks Employing Cooperative Multi-hop Transmission With and Without Presence of Hardware Impairments" Entropy, 21(2), 217, 2019. https://doi.org/10.3390/e21020217

[43] P. T. Tin, P. M. Nam, T. T. Duy, T. T. Phuong, M. Voznak, "Secrecy Performance of TAS/SC-based Multi-hop Harvest-to-Transmit Cognitive WSNs under Joint Constraint of Interference and Hardware Imperfection" Sensors, 19(5), 1160, 2019. https://doi.org/10.3390/s19051160

[44] P. M. Nam, P. V. Ca, T. T. Duy, Khoa N. Le, "Secrecy Performance Enhancement Using Path Selection Over Cluster-Based Cognitive Radio Networks" in 5th EAI International Conference on Industrial Networks and Intelligent Systems, HoChiMinh city, Vietnam, 2019. https://doi.org/10.1007/978-3-030-30149-1_6 